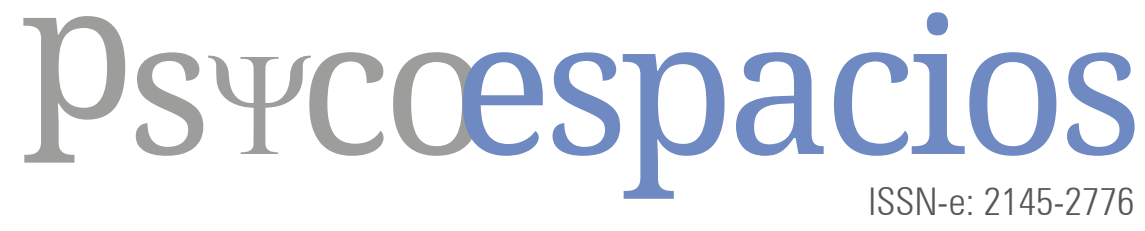

Vol. 15, № 26, enero-junio 2021 


\section{Fortalezas para la prevención de conductas de riesgo en adolescentes del Distrito de Aguablanca de Cali (Colombia)}

Strengths for the prevention of risk behaviors in adolescents in Distrito de Aguablanca of Cali (Colombia)

Norman Darío Moreno-Carmona ${ }^{\text {A }}$

Universidad San Buenaventura, Colombia https://orcid.org/0000-0002-8216-2569

Mayelli Tasamá Rincón

Casa de Justicia de Aguablanca, Secretaría de Seguridad y Justicia, Colombia https://orcid.org/0000-0002-2164-0432

Carlos Alberto Rojas Cruz Secretaría de Seguridad y Justicia del Municipio de Santiago de Cali, Colombia https://orcid.org/0000-0003-0915-6653

José Darío Soto Soto

Corporación Juan Bosco, Colombia https://orcid.org/0000-0002-2277-9989

${ }^{a}$ Autor de correspondencia: norman.moreno@usbmed.edu.co

Recibido: 4 mayo 2021 • Aceptado: 6 agosto 2021 • Publicado: 29 agosto 2021

Cómo citar este artículo: Moreno-Carmona, N.D., Tasamá Rincón, M., Rojas Cruz, C.A. y Soto Soto, J.C. (2021). Fortalezas para la prevención de conductas de riesgo en adolescentes del Distrito de Aguablanca de Cali (Colombia). Psicoespacios, 15(26). http///doi 


\section{Resumen}

El propósito de este artículo es presentar los resultados de una investigación ${ }^{1}$ acerca de la relación entre las fortalezas y la presencia de conductas de riesgo de los adolescentes atendidos en los diversos programas de la Corporación Juan Bosco de la ciudad de Cali, Colombia. Tomando como base la perspectiva del desarrollo positivo, se realizó un estudio correlacional comparando 188 adolescentes de la Corporación, clasificados en dos grupos según su tiempo de vinculación. Asimismo, se seleccionó otro grupo conformado por 81 adolescentes del sector, no vinculados a la Corporación Juan Bosco. Se utilizaron los instrumentos de fortalezas y factores de protección adaptados para México y Colombia; y para las conductas de riesgo se utilizó el inventario de conductas de riesgo de Andrade Palos y Betancourt Ocampo (2008). Los resultados demostraron que las fortalezas externas relacionadas con las figuras parentales y los pares resultan más decisivas para que los adolescentes no se involucren en conductas de riesgo.

Palabras claves: adolescencia, desarrollo positivo, conductas de riesgo, fortalezas.

\section{Abstract}

The purpose of this article is to present the results of a research on the relationship between the strengths and the presence of risk behaviors of adolescents served in the various programs of the Juan Bosco Corporation in the city of Cali, Colombia. Based on the positive youth development perspective, a correlational study was conducted comparing 188 adolescents of the Corporation, classified in two groups according to their time of association. Another group of 81 adolescents from the sector, not linked to the Juan Bosco Corporation, was selected. Strengths and protective factors adapted for Mexico and Colombia were used; and risk behaviors were using the risk behavior inventory of Andrade Palos and Betancourt Ocampo (2008). The results showed that external strengths related to parental figures and peers are more decisive for adolescents not to engage in risky behaviors.

Key Words: Adolescence, Positive Development, Risk Behaviors, Strenghts.

\section{Introducción}

Una de las particularidades de la experiencia desarrollada durante más de 25 años por la Corporación Juan Bosco, principalmente en la ciudad de Cali, es que se propone una prevención a partir de estrategias pedagógicas por fuera del claustro escolar, ya sea con jóvenes que han desertado del sistema formal o con otros que, si bien permanecen en él, encuentran en la calle su espacio de socialización por excelencia (Moreno Carmona et al., 2007).

Aspectos como el afecto y la confianza, la amabilidad y la norma, partir de las potencialidades y no de las carencias de los jóvenes son algunos principios rectores de su propuesta educativa. Esto se explicita en sus principios pedagógicos: educar en positivo, ganar el corazón y hacer crecer desde dentro (Moreno Carmona et al., 2007).

Dicha perspectiva parece articularse, implícitamente, con la del denominado desarrollo positivo en adolescentes (Positive Youth Development, PYD) que desde la psicología se ha venido abordando en las últimas décadas. El PYD no niega la presencia de la violencia, el consumo de drogas o las prácticas sexuales de riesgo, sino que centra su mirada principalmente en las habilidades, conductas y competencias que, junto con la existencia de comportamientos saludables, permitan

\footnotetext{
1 La investigación se desarrolló en el año 2018 y fue financiada por un convenio entre la Universidad de San Buenaventura, Medellín y la Corporación Juan Bosco.
} 
obtener el éxito en diferentes ámbitos como el psicológico, académico y familiar (Oliva et al., 2010).

El PYD propone una serie de fortalezas externas: familia, escuela, pares, que hacen referencia a un clima afectuoso y seguro; y fortalezas internas, entendidas como las características psicológicas de los jóvenes, como por ejemplo: autoestima, resiliencia, responsabilidad personal, expectativas de futuro, capacidad para tomar decisiones, entre otras (Oliva Delgado et al., 2011).

Estudios previos aseguran que a mayor número de fortalezas, menor involucramiento en conductas de riesgo (Andrade Palos et al., 2017; Benson et al., 1998; Dunn et al., 2011; Lenzi et al., 2015; Moreno-Carmona et al., 2018; Moreno-Carmona y Alvis-Rizzo, 2018; Murphey et al., 2004; Oman et al., 2004).

Después de 25 años de trabajo ininterrumpido con jóvenes de la ciudad de Cali y otras ciudades del suroccidente colombiano, y habiendo hecho unos primeros acercamientos a la perspectiva teórica de la PYD, la Corporación Juan Bosco quiso recurrir a las herramientas metodológicas que este modelo ofrece (escalas de medición de fortalezas y recursos) para dar cuenta de la manera como su intervención sigue siendo pertinente en la prevención y promoción de los habitantes de sectores vulnerables del país, desde una visión positiva de los adolescentes.

El interés en las conductas de riesgo y en la prevención ha sido también focalizado en las manifestaciones que de dichas problemáticas se presentan en la población que atiende la Corporación Juan Bosco. Por ese motivo, iniciativas como el programa Odisea, las Casas Juveniles y la formación artístico-cultural son estrategias que han sido desarrolladas para tratar el creciente fenómeno del consumo de alcohol y otras sustancias psicoactivas, el involucramiento de los adolescentes en pandillas o grupos delincuen- ciales, así como diversas conductas de riesgo entre la población atendida. (Moreno Carmona et al., 2007).

Catalano et al. (2004), así como Parra Jiménez et al. (2009), al realizar sendas revisiones sobre programas enfocados en la PYD, encontraron que la mayoría de ellos obtuvieron cambios positivos en el comportamiento de los jóvenes, incluyendo mejoras importantes en las habilidades interpersonales, la calidad de las relaciones con sus compañeros y los adultos, y el desarrollo de un mayor compromiso social cuando son adultos, así como mejores niveles educativos y ocupacionales. Igualmente, Benson et al. (2006) afirman que fortalezas como relaciones clave, oportunidades, valores, habilidades y autopercepciones ayudan a los jóvenes a limitar su involucramiento en conductas de riesgo.

Recientemente, los trabajos de Martín García (2014) y Habib et al. (2014) señalan los beneficios que tiene para adolescentes en situaciones de vulnerabilidad el que participen en proyectos comunitarios, mediante el desarrollo de algunas prácticas educativas para el fortalecimiento de valores, competencias y conocimientos que les permitan superar los riesgos de exclusión, aprender roles y competencias sociales.

Más específicamente, en Colombia se ha encontrado que los adolescentes que nunca han intentado suicidarse puntúan más alto en fortalezas como apoyo y supervisión de la madre y el padre, importancia de la salud y toma de decisiones (Moreno-Carmona et al., 2018); también, que los jóvenes con menos conductas sexuales riesgosas cuentan con un mayor número de fortalezas externas (Moreno-Carmona y Alvis-Rizzo, 2018).

Es tal coincidencia en la PYD la que convoca un proyecto de investigación que, utilizando herramientas desde esta perspectiva, procure dar cuenta de la presencia de fortalezas en los jóvenes atendidos actualmente 
en la institución y su posible vínculo con la prevención de conductas de riesgo.

\section{Participantes}

Se realizó un estudio correlacional comparativo entre un grupo de jóvenes que asisten a los distintos programas desarrollados por la Corporación Juan Bosco (CJB) y otro grupo del mismo sector, pero que no está vinculado a ninguna actividad desarrollada por la institución.

La muestra fue no probabilística, intencional y guiada por el interés manifiesto de los adolescentes por participar del estudio. Estuvo compuesta, en primer lugar, por 188 adolescentes de la CJB, hombres y mujeres, 74 que están recién vinculados a la institución (menos de ocho meses en el proceso) y 114 que llevan ocho meses o más. Y, en segundo lugar, se seleccionó otro grupo conformado por 81 adolescentes del sector, no vinculados a la Corporación Juan Bosco.

Debido a que se trata de evaluar el impacto de la intervención de la СJB para prevenir conductas de riesgo mediante el desarrollo de fortalezas, y dado que el tiempo promedio establecido por la entidad estatal contratante (Instituto Colombiano de Bienestar Familiar, ICBF) para que cada joven permanezca vinculado a los programas de la institución es de un año, los mismos educadores y profesionales de la institución consideraron que un tiempo prudente para comenzar a ver dicho impacto serían los ocho meses de permanencia.

\section{Instrumentos}

Se utilizaron los instrumentos de fortalezas y factores de protección adaptados para México por Andrade et al. (2013), y para Colombia por Andrade et al. (2017) y Moreno-Carmona et al. (2018). Se trata de escalas tipo Likert con cuatro opciones de respuesta, que van de
$1=$ Ninguno a $4=$ Todos, de $1=$ Nunca a $4=$ Siempre, de $1=$ Nada importante a $4=$ Muy importante o de 1 = Totalmente en desacuerdo a $4=$ Totalmente de acuerdo.

Las variables consideradas en el estudio son las fortalezas externas e internas y algunas conductas de riesgo. Las fortalezas externas hacen referencia a las oportunidades de desarrollo positivo que se dan a través de las múltiples interacciones adultos-adolescente-pares y son reforzadas por las instituciones, como la escuela y la comunidad. Para este estudio se evaluaron la familia (la comunicación y supervisión de ambos padres) y la influencia de los pares (amigos involucrados en pandillas y conductas de riesgo, y amigos saludables). Las fortalezas internas se refieren a valores, habilidades y autopercepciones que los jóvenes desarrollan para regularse a sí mismos. Se evaluaron la responsabilidad, la importancia de la salud, la toma de decisiones, la resistencia a la presión de los pares, la visión de futuro, el bienestar psicológico, la autoestima y la conducta prosocial.

Para las conductas de riesgo se utilizó el inventario de Conductas de Riesgo de Andrade Palos y Betancourt Ocampo (2008) y Moreno Carmona (2014). Mediante autoreporte se evaluaron específicamente cuatro tipos de conducta: (1) consumo de psicoactivos, que evalúa la frecuencia y cantidad del consumo de cigarrillos, sustancias psicoactivas, así como la edad del primer consumo; (2) conductas sexuales de riesgo: el inicio temprano de las relaciones sexuales, el uso de métodos anticonceptivos, el tipo de parejas sexuales y la frecuencia de uso del condón; (3) conductas contraventoras: distintos tipos de infracciones a la ley y su frecuencia y (4) el intento de suicidio: evalúa si el adolescente ha intentado suicidarse una o varias veces, así como los motivos que lo llevaron a hacerlo y los métodos utilizados.

Los instrumentos, diseñados inicialmente para adolescentes mexicanos, ya ha- 
bían sido adaptados al contexto colombiano obteniendo índices de confiabilidad similares (Alfa de Cronbach superiores a 0,86 en las fortalezas externas y superiores a 0,64 para fortalezas internas) (Andrade et al., 2017).

\section{Procedimiento}

Se diligenció un consentimiento firmado por los padres, por lo que la selección estuvo sujeta tanto a la obtención del consentimiento de los padres o autorización de las autoridades competentes (Instituto Colombiano de Bienestar Familiar o jueces de infancia y adolescencia), así como a la disposición voluntaria de los adolescentes para participar. Los instrumentos fueron aplicados individualmente, utilizando un formulario digital diseñado en Microsoft Access, en los computadores disponibles en las casas juveniles de la Corporación Juan Bosco, y la base de datos de Excel fue exportada a SPSS-25 donde finalmente se hicieron los análisis estadísticos no paramétricos, debido a que las variables no tuvieron distribución normal.

\section{Resultados}

Inicialmente se describen las características sociodemográficas de los participantes (tabla 1). En el grupo de la CJB el número de hombres y mujeres es similar, mientras que en el grupo externo (GE) predominan las mujeres. Las edades de todos los participantes oscilan entre 14 y 17 años $(M=15,6-D E=1,72)$. Aunque la mayoría son solteros y estudiantes, en la muestra de la CJB aparece un mayor número de adolescentes que ya trabajan y que han conformado relaciones de pareja.
Tabla 1. Características sociodemográficas de los participantes

\begin{tabular}{cccccc}
\hline & & \multicolumn{2}{c}{ CJB } & \multicolumn{2}{c}{ GE } \\
& & (n = 188) & \multicolumn{2}{c}{$(\mathbf{n}=\mathbf{8 1})$} \\
\hline Característica & $\mathrm{n}$ & $\%$ & $\mathrm{n}$ & $\%$ \\
\hline Sexo & & & & & \\
\hline & Hombres & 93 & 49,5 & 32 & 39,5 \\
& Mujeres & 94 & 50,0 & 49 & 60,5 \\
Edad* & N.R. & 1 & 0,5 & & \\
\hline & & & & & \\
\hline 13 o menos & 27 & 14,4 & 8 & 12,1 \\
14 & 34 & 18,1 & 11 & 12,1 \\
15 & 37 & 19,7 & 18 & 21,2 \\
16 & 27 & 14,4 & 15 & 13,6 \\
17 & 42 & 22,3 & 20 & 28,8 \\
18 o más & 20 & 10,7 & 9 & 12,1 \\
N.R. & 1 & 0,5 & & \\
\hline
\end{tabular}

\section{Estado civil}

$\begin{array}{ccccc}\text { Soltero } & 178 & 94,7 & 72 & 95,1 \\ \text { Unión libre } & 7 & 3,7 & 3 & 3,7 \\ \text { Casado } & 2 & 1,1 & 1 & 1,2 \\ \text { N.R. } & 1 & 0,5 & & \end{array}$

Ocupación

$\begin{array}{ccccc}\text { Estudia } & 153 & 81,4 & 72 & 88,9 \\ \text { Trabaja } & 11 & 5,9 & 2 & 2,5 \\ \text { udia y trabaja } & 9 & 4,8 & 2 & 2,5 \\ \text { tudia ni trabaja } & 14 & 7,4 & 5 & 6,2 \\ \text { N.R. } & 1 & 0,5 & & \end{array}$

\section{Tipo de familia}

\begin{tabular}{ccccc}
\hline Biparental & 81 & 43,1 & 33 & 41,3 \\
Monoparental materna & 53 & 28,2 & 29 & 35,8 \\
Monoparental paterna & 7 & 3,7 & 2 & 2,5 \\
Recompuesto padrastro & 28 & 14,9 & 8 & 9,9 \\
Recompuesta madrastra & 4 & 2,1 & 2 & 2,5 \\
Familia extensa (sin padres) & 11 & 5,9 & 6 & 7,4 \\
N.R. & 4 & 2,1 & 1 & 1,2 \\
\hline
\end{tabular}

*Media y desviación estándar de la edad en cada grupo: (CJB) $\mathrm{M}=15,5 ; \mathrm{DE}=1,77$ (GE) $\mathrm{M}=15,7$; $\mathrm{DE}=1,68$ 
Asimismo, es de resaltar que, si bien en ambos grupos la mayoría tiene familia biparental, esta tipología no alcanza el $50 \%$ de la muestra y es seguida por la familia monoparental materna y recompuesta con padrastro.

Con respecto a las conductas sexuales de riesgo, hay un mayor porcentaje de adolescentes que no han iniciado su actividad sexual ( $\mathrm{CJB}=55,3 \%$ y GE $=51,9 \%)$ y la mayor parte de los que ya lo hicieron tuvieron su primera relación sexual antes de los 16 años; alrededor del $40 \%$ de ellos iniciaron antes de los 14 años. Teniendo en cuenta que un 20,5 $\%$ de los adolescentes de la сјв у 23,7 \% del grupo control señalan que su primera pareja sexual fue una persona mayor de edad, es posible que en algunos casos se haya tipificado el abuso.

Si bien el mayor porcentaje reporta haber tenido su primera relación sexual con su novia(o) o un amigo(a), un $14,4 \%$ de la СJB y $10,3 \%$ del GE refieren una pareja ocasional u otra persona. El 63,9 \% de la CJB y el 51,3 $\%$ del GE mantienen actividad sexual actualmente, en su mayoría con el novio(a) o un amigo(a), manteniéndose el porcentaje de parejas ocasionales en algunos (10\%).

Alrededor de un $60 \%$ de ambos grupos manifiesta haber usado anticonceptivo en su primera relación sexual y todavía hay un porcentaje importante de ellos que no usa anticonceptivos en sus relaciones sexuales actuales $(\mathrm{CJB}=18,2 \%$ y GE $=32,9 \%)$. Así mismo, 49 \% de los adolescentes de la institución dicen usar condón siempre, mientras en el grupo control ese porcentaje es del 22,2\%. Preocupa el $17,7 \%$ de la CJB y el $44,4 \%$ del GE que dicen usarlo rara vez o nunca.

Con respecto al consumo de tabaco (tabla 2) el mayor porcentaje de los adolescentes de ambos grupos nunca ha fumado, y de los que sí lo ha hecho, la mayoría lo hizo por primera vez antes de los 15 años. Gran parte de los que fuman actualmente pertenecen a la CJB.

Tabla 2. Consumo de cigarrillo e intento de suicidio

\begin{tabular}{ccccc}
\hline & \multicolumn{2}{c}{ CJB } & \multicolumn{2}{c}{ GE } \\
& $(\mathbf{n}=\mathbf{1 8 8})$ & \multicolumn{1}{c}{$(\mathbf{n}=\mathbf{6 6})$} \\
\hline Característica & $\mathbf{n}$ & $\mathbf{\%}$ & $\mathbf{n}$ & $\mathbf{\%}$ \\
\hline Has fumado cigarrillo alguna vez & & \\
\hline Sí & 46 & 24,5 & 14 & 17,3 \\
No & 141 & 75,0 & 66 & 81,5 \\
N.R. & 1 & 0,5 & 1 & 1,2 \\
\hline Edad primer cigarrillo* & & & & \\
\hline 12 o menos & 19 & 42,2 & 5 & 35,7 \\
$13-14$ & 14 & 31,1 & 6 & 42,9 \\
$15-16$ & 11 & 24,4 & 3 & 21,4 \\
17 o más & 1 & 2,2 & & \\
\hline
\end{tabular}

\section{Ha fumado en el último mes}

Sí

No

Intento de suicidio

Una vez

Más de una vez

No

N.R.

$\begin{array}{cccc}13 & 56,5 & 2 & 14,3 \\ 10 & 43,5 & 12 & 85,7\end{array}$

$23 \quad 12,2 \quad 13 \quad 16,0$

$\begin{array}{llll}7 & 3,7 & 4 & 4,9\end{array}$
$157 \quad 83,5 \quad 64 \quad 79,0$

10,5

Edad primer intento de suicidio ${ }^{\text {*t* }}$

\begin{tabular}{ccccc} 
Menos de 13 & 10 & 33,3 & 3 & 17,6 \\
$13-14$ & 16 & 53,3 & 11 & 64,7 \\
$15-17$ & 4 & 13,3 & 3 & 17,7 \\
\hline
\end{tabular}

* Media y desviación estándar de la edad de primer consumo de cigarrillo en cada grupo: (СЈB) $\mathrm{M}=$ 12,$1 ; \mathrm{DE}=3,9$ (GE) $\mathrm{M}=12,9 ; \mathrm{DE}=1,75$

** Media y desviación estándar de la edad del primer intento de suicidio en cada grupo: (слB) $\mathrm{M}=$ 12,$2 ; \mathrm{DE}=3,9$ (GE) $\mathrm{M}=13,5 ; \mathrm{DE}=1,59$

Con referencia al intento de suicidio, el $15,9 \%$ de la сјв у $20,9 \%$ del GE lo han intentado al menos una vez y en su mayoría antes de los 15 años.

Para el comparativo de fortalezas y teniendo en cuenta el tiempo de permanencia de los adolescentes en la Corporación Juan Bosco, se consideraron tres grupos: los que llevaban menos de 8 meses, los que llevaban 8 meses o más y el grupo externo. Al compa- 
rar la presencia de Fortalezas Externas, Internas y Fortalezas en general en los tres grupos de adolescentes, el análisis de varianza (ANOVA) no evidenció diferencias estadísticamente significativas entre los grupos.

También se procedió a correlacionar cada una de las fortalezas con los índices de consumo de psicoactivos y conductas con- traventoras, encontrando algunas relaciones significativas (tabla 3). Dichos índices se calcularon, en el caso del consumo de drogas, al asignar un valor diferencial de acuerdo con el tipo de sustancia (de blanda a dura) multiplicado por la frecuencia de su consumo; y en el caso de las contravenciones, asignando un valor, dependiendo de la gravedad de la infracción multiplicada por la frecuencia.

Tabla 3. Correlación de Spearman entre fortalezas-conductas de riesgo según vinculación a СJB

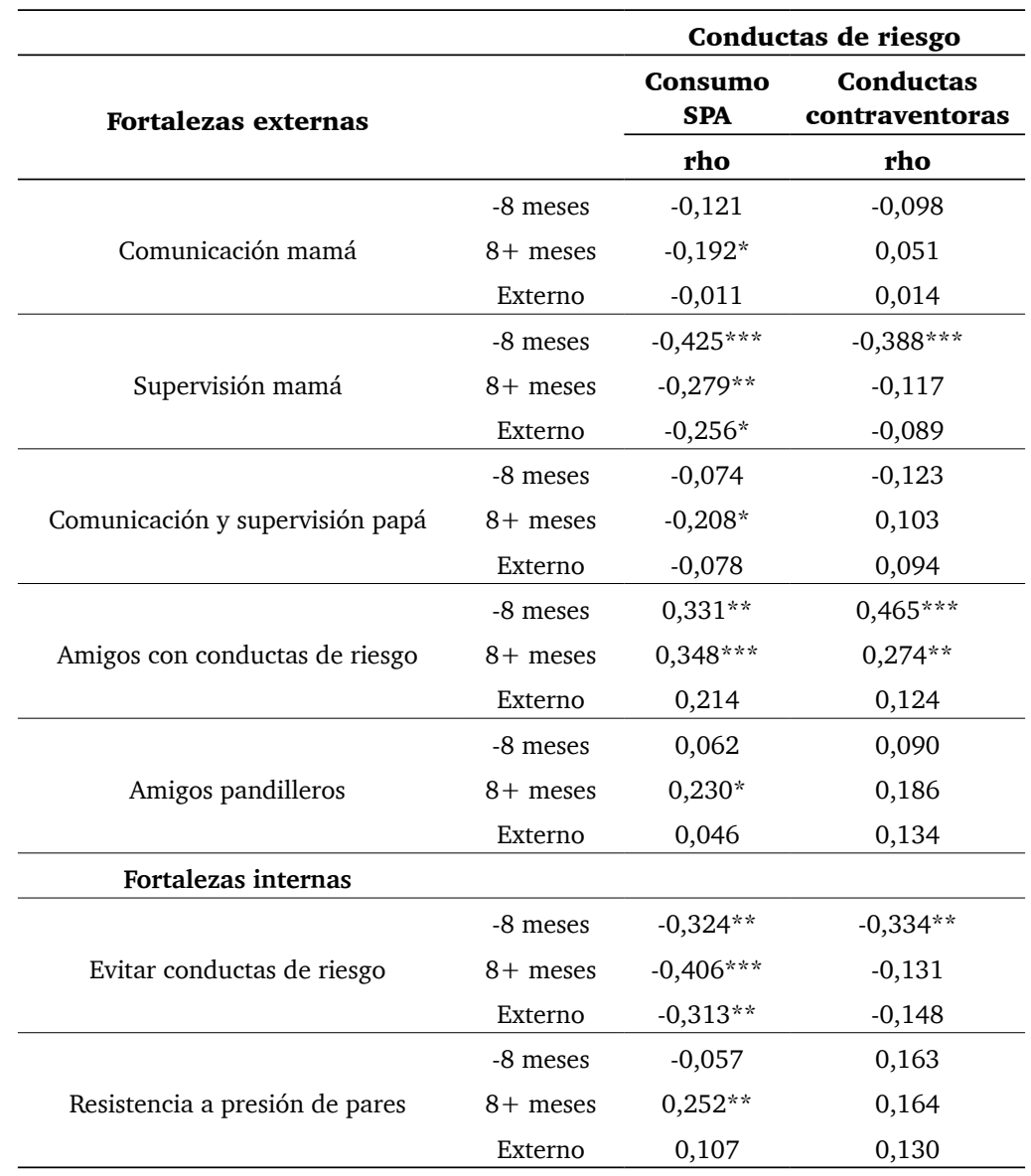

Nota: SPA $=$ Sustancias Psicoactivas

$* \mathrm{p} \leq 0,05 \quad * * \mathrm{p} \leq 0,01 \quad * * * \mathrm{p} \leq 0,001$ 
Entre los adolescentes de la CJB que llevan más tiempo en la institución (ocho meses o más) se encontraron correlaciones bajas de la mayor parte de las fortalezas con el consumo de psicoactivos, obteniendo los puntajes más altos en la evitación de conductas de riesgo. Y con respecto a las conductas contraventoras se evidencian algunas correlaciones también bajas con la supervisión materna, amigos con conductas de riesgo y evitación de conductas de riesgo en los grupos vinculados a la CJB.

Lo que se puede observar en la tabla 3 es que la supervisión materna, el tener amigos con conductas de riesgo y la evitación de dichas conductas están más asociadas al bajo involucramiento de los adolescentes en el consumo de drogas y conductas contraventoras.

Para calcular los puntajes totales de las fortalezas, se obtuvo el percentil 75 de cada una de ellas, de tal manera que, en cuanto a los jóvenes que estuvieran dentro de este, se consideró que la presencia de dicha fortaleza evaluada es sobresaliente y en cuanto a los que estuvieran por debajo de ese percentil se consideró que no es tan característica de ellos. Posteriormente, ya con la identificación de las fortalezas, se procedió a sumar aquellas que alcanzaron el percentil 75 en cada participante; en la tabla 4 puede observarse la distribución del número de fortalezas en los tres grupos poblacionales. Se encontró que una mayor proporción de adolescentes presentaron de una a dos fortalezas externas y de una a dos fortalezas en general, mientras en los tres grupos la mayoría registraron cero fortalezas internas y ninguno presenta cuatro o más, siendo los adolescentes vinculados a la institución quienes presentan mayores porcentajes de fortalezas internas.
Tabla 4. Distribución de los participantes por número de fortalezas y por grupo

\begin{tabular}{|c|c|c|c|}
\hline $\begin{array}{c}\text { Fortalezas } \\
\text { externas }\end{array}$ & $\begin{array}{c}\text { Menos de } \\
8 \text { meses en } \\
\text { la cJB } \\
(n=74) \\
\% \\
\end{array}$ & $\begin{array}{c}8 \text { meses } \\
\text { o más en } \\
\text { la cJB } \\
(n=114) \\
\%\end{array}$ & $\begin{array}{c}\text { Grupo } \\
\text { externo } \\
(0 \text { meses }) \\
(\mathrm{n}=81) \\
\%\end{array}$ \\
\hline Cero & 27,0 & 44,7 & 29,6 \\
\hline Una & 21,6 & 21,9 & 28,4 \\
\hline Dos & 21,6 & 11,4 & 18,5 \\
\hline Tres & 8,1 & 7,9 & 12,3 \\
\hline Cuatro & 9,5 & 9,6 & 2,5 \\
\hline Más de cuatro & 12,2 & 4,4 & 8,6 \\
\hline \multicolumn{4}{|c|}{ Fortalezas internas } \\
\hline Cero & 58,1 & 55,3 & 61,7 \\
\hline Una & 28,4 & 34,2 & 32,1 \\
\hline Dos & 12,2 & 10,5 & 6,2 \\
\hline Tres & 1,4 & & \\
\hline \multicolumn{4}{|l|}{$\begin{array}{l}\text { Total de } \\
\text { fortalezas }\end{array}$} \\
\hline Cero & 16,2 & 27,2 & 22,2 \\
\hline Una & 17,6 & 30,7 & 25,9 \\
\hline Dos & 29,7 & 13,2 & 19,8 \\
\hline Tres & 12,2 & 9,6 & 13,6 \\
\hline Cuatro & 6,8 & 8,8 & 8,6 \\
\hline Más de cuatro & 17,6 & 10,5 & 9,9 \\
\hline
\end{tabular}

Utilizando el chi cuadrado para analizar la asociación entre las conductas de riesgo (intento de suicidio, inicio temprano de relaciones sexuales y uso de condón) y las fortalezas (percentil 75) se encontraron asociaciones significativas en la muestra total entre el intento de suicidio con la comunicación materna $\left(\mathrm{x}^{2}=5,29 \mathrm{p} \leq 0,05\right)$, con la comunicación y supervisión paterna $\left(\mathrm{x}^{2}\right.$ $=4,09 \mathrm{p} \leq 0,05)$, los amigos con conductas de riesgo $\left(\mathrm{x}^{2}=6,14 \mathrm{p} \leq 0,05\right)$, los amigos pandilleros $\left(\mathrm{x}^{2}=7,93 \mathrm{p} \leq 0,01\right)$ los amigos saludables $\left(\mathrm{x}^{2}=4,53 \mathrm{p} \leq 0,05\right)$ y con la toma de decisiones $\left(\mathrm{x}^{2}=5,92 \mathrm{p} \leq 0,05\right)$; también se hallaron relaciones significativas entre el inicio temprano de relaciones sexuales con la 
supervisión materna $\left(\mathrm{x}^{2}=5,28 \mathrm{p} \leq 0,05\right)$, los amigos con conductas de riesgo $\left(\mathrm{x}^{2}=7,59 \mathrm{p}\right.$ $\leq 0,01)$ y la resistencia a presión de pares $\left(\mathrm{x}^{2}\right.$ $=6,09 \mathrm{p} \leq 0,05)$. Finalmente, se encontraron asociaciones entre el uso de condón y el tener amigos con conductas de riesgo $\left(\mathrm{x}^{2}=\right.$ $4,43 \mathrm{p} \leq 0,05)$.

En los grupos vinculados a la CJB, específicamente en los recién vinculados existe asociación entre la comunicación materna y el intento de suicidio $\left(\mathrm{x}^{2}=4,89 \mathrm{p} \leq 0,05\right)$, también existe asociación entre tener amigos con conductas de riesgo y el inicio temprano de relaciones sexuales (antes de los 14 años) $\left(x^{2}=9,82 \mathrm{p} \leq 0,01\right)$ y asociación entre la resistencia a la presión de pares y el uso de condón $\left(\mathrm{x}^{2}=7,11 \mathrm{p} \leq 0,01\right)$; mientras que en los adolescentes que llevan más de ocho meses vinculados a los programas de la institución se observó relación significativa entre toma de decisiones e intento de suicidio $\left(\mathrm{x}^{2}\right.$ $=3,83 \mathrm{p} \leq 0,05)$. En el grupo externo solo se observó una asociación significativa entre el tener amigos saludables y el uso del condón $\left(\mathrm{x}^{2}=4,62 \mathrm{p} \leq 0,05\right)$.

También se analizó, mediante correlación de Spearman, la asociación entre el número de fortalezas internas, externas y fortalezas en general con el consumo de psicoactivos y las contravenciones, encontrando únicamente relación significativa baja entre el número de fortalezas externas y el índice de consumo de drogas en el total de participantes (rho $=-0,172 \mathrm{p} \leq 0,01$ ) y en el grupo de adolescentes con más tiempo de vinculación a la СJB $(r h o=-0,228 \mathrm{p} \leq 0,05)$.

Además, se analizaron las diferencias en la presencia o no de conductas de riesgo según el número de fortalezas mediante chi cuadrado. Los datos indican que en la muestra total de participantes el intento de suicidio difiere de acuerdo con el número de fortalezas en general $\left(\mathrm{x}^{2}=22.87 \mathrm{p} \leq .01\right)$ y el número de fortalezas externas $\left(\mathrm{x}^{2}=18,43 \mathrm{p}\right.$ $\leq 0,01)$; y en el grupo de adolescentes con más tiempo de vinculación a la CJB, el inicio temprano de las relaciones sexuales difiere de acuerdo con el número de fortalezas internas $\left(x^{2}=7,27 \mathrm{p} \leq 0,05\right)$. Con respecto al grupo externo, también se encontraron diferencias en el intento de suicidio con relación al número de fortalezas en general $\left(\mathrm{x}^{2}=16,05 \mathrm{p}\right.$ $\leq 0,05)$.

Al evaluar las fortalezas relacionadas con el bienestar (crecimiento personal, relaciones positivas, propósito de vida, planes a futuro, control personal y autoestima) no se encontraron diferencias; sin embargo, como lo ilustra la tabla 5, se evidenciaron diferencias estadísticamente significativas en la prosocialidad de los adolescentes según estén vinculados o no a la cJB.

Tabla 5. Prosocialidad de los adolescentes según vinculación o no a la CJB

\begin{tabular}{lccccc}
\hline & $\begin{array}{c}\text { Grupo cJB } \\
(\mathbf{n}=\mathbf{1 8 6})\end{array}$ & \multicolumn{2}{c}{$\begin{array}{c}\text { Grupo } \\
\text { externo } \\
(\mathbf{n}=\mathbf{8 1})\end{array}$} & \\
\hline & $\mathbf{M}$ & $\mathbf{D E}$ & $\mathbf{M}$ & $\mathbf{D E}$ & $\mathbf{t}$ \\
\hline $\begin{array}{l}\text { Prosocialidad ayuda } \\
\text { emocional }\end{array}$ & 3,12 & 0,61 & 2,96 & 0,61 & $1,98^{*}$ \\
$\begin{array}{l}\text { Prosocialidad } \\
\text { reconocimiento }\end{array}$ & 2,16 & 0,87 & 1,89 & 0,78 & $2,40^{*}$ \\
$\begin{array}{l}\text { Prosocialidad } \\
\text { altruismo }\end{array}$ & 2,64 & 0,72 & 2,38 & 0,58 & $3,066^{* *}$ \\
$*$ p $\leq 0,05 * * \mathrm{p} \leq 0,01$ & & & & & \\
\hline
\end{tabular}

\section{Discusión}

Teniendo en cuenta los anteriores resultados se pueden identificar que, tal como lo mencionan Catalano et al. (2004), uno de los logros del trabajo desarrollado por la Corporación Juan Bosco es el fortalecimiento de las relaciones interpersonales de los niños, niñas y adolescentes que se han vinculado a los programas ofrecidos por esta ONG; cuestión que se evidencia en la construcción de vínculos sociales positivos con pares, que les permiten hacer frente a las conductas de riesgo 
relacionadas con la sexualidad y el suicidio. De igual manera, se encontraron diferencias en la prosocialidad de los adolescentes pertenecientes a la cJB y los externos, debido a que los primeros tienden a realizar más acciones altruistas y brindar ayuda emocional a otros.

Los anteriores hallazgos son un primer paso para identificar esos factores de desarrollo que permiten la prevención de conductas riesgosas como el consumo de drogas y el intento de suicidio (Benson et al., 2006). Y son principalmente las fortalezas externas relacionadas con las figuras parentales y los pares las que evidencian mayor asociación con la disminución de las conductas de riesgo.

El principal hallazgo es que los jóvenes con menos conductas de riesgo presentan más fortalezas que aquellos que presentan conductas más riesgosas. Esto confirma lo propuesto por autores que han utilizado el enfoque de fortalezas de la PYD (Andrade et al., 2017; Benson et al., 1998; Moreno-Carmona et al., 2018; Murphey et al., 2004), quienes encontraron un mayor número de fortalezas tanto internas como externas en los jóvenes que reportaron menor presencia de conductas de riesgo.

También se confirma lo encontrado por Dunn et al. (2011) y Oman et al. (2004) respecto a que los jóvenes que no han consumido sustancias tienen menos amigos que presentan conductas de riesgo.

Se evidencia que el desarrollo positivo promovido desde la CJB ha exaltado las relaciones positivas entre pares como un mecanismo que fomenta las fortalezas externas y empiezan a construir relaciones claves entre amigos que tiene como consecuencia el no involucramiento en conductas riesgosas; y aunque las fortalezas internas fueron las menos identificadas en los adolescentes participantes en la investigación, son los vinculados a la CJB quienes presentan mayor presencia de dichas fortalezas.
También se evidencia que educar en positivo hace que el niño, la niña y el adolescente puedan generar acciones más empáticas hacia sus pares, fomentando la prosocialidad.

El proyecto plantea la posibilidad de desarrollar estrategias que permitan el fortalecimiento de activos internos y externos en los niños y adolescentes, para la prevención del involucramiento en conductas de riesgo.

Dado que la principal limitación del estudio es la muestra, tanto por su tamaño como por su no aleatoriedad, es probable que, con muestras más representativas y estudios longitudinales, se puedan obtener resultados más contundentes. Así mismo, es recomendable diseñar y poner a prueba programas implementados bajo la perspectiva de la PYD, que permitan diseños pre-post con resultados más contundentes.

\section{Referencias}

Andrade Palos, P. y Betancourt Ocampo, D. (2008). Factores individuales, familiares y sociales y conductas de riesgo en adolescentes. En P. Andrade Palos, J. L. Cañas Martínez y D. Betancourt Ocampo (Comp.), Investigaciones psicosociales en adolescentes (pp. 181-213). Unicach.

Andrade Palos, P., Betancourt Ocampo, D., Moreno Carmona, N. D. y Alvis Rizzo, A. (2017). Fortalezas externas desde el modelo de desarrollo positivo de los jóvenes y consumo de sustancias en una muestra de adolescentes mexicanos y colombianos. Avances en Psicología Latinoamericana, 35(3), 515-529. https://doi.org/10.12804/revistas.urosario. edu.co/apl/a.4095

Andrade, P.P., Morales, N., Sánchez, X. C. O. y González, G. A. (2013). Fortalezas de los jóvenes que no presentan conductas de riesgo. El Psicólogo Anáhuac, 16, 17-24.

Benson, P. L., Leffert, N., Scales, P. C. y Blyth, D. A. (1998). Beyond the "village" rhetoric: Creating healthy communities for children and adolescents. Applied Developmental Science, 
2(3), 138-159. https://doi.org/10.1207/ s1532480xads0203_3

Benson, P. L., Scales, P. C., Hamilton, S. F. y Sesma Jr., A. (2006). Positive youth development: theory, research and applications. En W. Damon y R. M. Lerner (Eds.), Handbook of Child Psychology (pp. 894-941). John Wiley \& Sons, Inc. https://doi. org/10.1002/9780470147658.chpsy0116.

Catalano, R. F., Berglund, M. L., Ryan, J. A. M., Heather, S., Lonczak, H. S. y Hawkins, J. D. (2004). Positive youth development in the United States: research findings on evaluations of positive youth development programs. The ANNALS of the American Academy of Political and Social Science, 591(1), 98-124. https:// doi.org/10.1177/0002716203260102

Dunn, M. S., Kitts, C., Lewis, S., Goodrow, B. y Scherzer, G. D. (2011). Effects of youth assets on adolescent alcohol, tobacco, marijuana use, and sexual behavior. Journal of Alcohol and Drug Education, 55(3), 23-40. https:// eric.ed.gov/?id=EJ954689

Habib, E. L., Zimmerman, M. A. y Ostaszewski, K. (2014). International note: Prevailing with extracurricular activities in an alcoholdominated environment: Sex differences in resilience among middle school youth. Journal of Adolescence, 37(6), 901-904. https://doi. org/10.1016/j.adolescence.2014.06.004

Lenzi, M., Dougherty, D., Furlong, M. J., Sharkey, J. y Dowdy, E. (2015). The configuration protective model: factors associated with adolescent behavioral and emotional problems. Journal of Applied Developmental Psychology, 38, 49-59. https://doi. org/10.1016/j.appdev.2015.03.003

Martín García, X. (2014). Adolescentes en riesgo y servicio a la comunidad. Bordón. Revista de Pedagogía, 66(3), 75-89. https://recyt. fecyt.es/index.php/BORDON/article/view/ Bordon.2014.66305

Moreno Carmona, N. D. (2014). Factores familiares y psicosociales asociados a problemas internalizados y externalizados en adolescentes colombianos [tesis doctoral, Universidad Iberoamericana]. Repositorio Institucional Ibero. http://ri.ibero.mx/handle/ibero/950
Moreno-Carmona, N. D. y Alvis-Rizzo, A. (2018). Fortalezas externas y conducta sexual en adolescentes colombianos. Revista Psicología Social y Personalidad, 34(1), 69-84.

Moreno-Carmona, N. D., Andrade-Palos, P. y Betancourt-Ocampo, D. (2018). Fortalezas en adolescentes de Colombia y México que nunca han intentado suicidarse. Revista Latinoamericana de Ciencias Sociales, Niñez y Juventud, 16(2), 797-807. https://doi. org/10.11600/1692715x.16210

Moreno Carmona, N. D., Chilito Ordoñez. E. y Trujillo Ceballos, J. O. (2007). No con golpes: educando en clave de afecto. Juan Bosco Corporación.

Murphey, D. A., Lamonda, K. H., Carney, J. K. y Duncan, P. (2004). Relationships of a brief measure of youth assets to health-promoting and risk behaviors. Journal of Adolescent Health, 34(3), 184-191. https://doi. org/10.1016/j.jadohealth.2003.05.004

Oliva, A., Ríos, M., Antolín, L., Parra, A., Hernando, A. y Pertegal, M. Á. (2010). Más allá del déficit: construyendo un modelo de desarrollo positivo adolescente. Infancia $y$ Aprendizaje, 33(2), 223-234. https://doi. org/10.1174/021037010791114562

Oliva Delgado, A., Reina, M., Hernando, Á., Antolín, L., Pertegal, M. Á., Parra, Á., Ríos, M., Estévez R. M. y Pascual, D. M. (2011). Activos para el desarrollo positivo y la salud mental en la adolescencia. Junta de Andalucía. Consejería de Salud. http://www.codajic.org/sites/ www.codajic.org/files/desarrolloPositivo activos.pdf

Oman, R. F., Vesely, S., Aspy, C. B., McLeroy, K. R., Rodine, S. y Marshall, L. (2004). The potential protective effect of youth assets on adolescent alcohol and drug use. American Journal of Public Health, 94(8), 1425-1430. https://doi.org/10.2105/AJPH.94.8.1425

Parra Jiménez, Á., Oliva Delgado, A., y Antolín Suárez, L. (2009). Los programas extraescolares como recurso para fomentar el desarrollo positivo adolescente. Papeles del Psicólogo, 30(3), 265-275. http://www. papelesdelpsicologo.es/pdf/1755.pdf 